\title{
Painful and Painless Constipation: All Roads Lead to (A Change in) Rome
}

\author{
Adil E. Bharucha ${ }^{1} \cdot$ Mayank Sharma ${ }^{1}$
}

Published online: 21 March 2018

(c) Springer Science+Business Media, LLC, part of Springer Nature 2018

Disease classifications are useful to the extent that they facilitate an understanding of the pathophysiology, guide the diagnosis or treatment, or predict the natural history of the condition. In the field of gastroenterology (GI), disease classifications have long been used for functional syndromes that have largely eluded diagnosis based on conventional criteria such as histological, serological, or biochemical data. The Rome criteria classify chronic constipation as: functional constipation (FC), constipation-predominant IBS (IBS-C), and defecatory disorder (DD). FC is defined by the presence of two or more of six bowel symptoms such as excessive straining to defecate. IBS-C is defined by abdominal pain that is associated, in time, with bowel disturbances (harder or less frequent stools) and/or relief of pain with defecation. DD, which is defined by symptoms of FC or IBS-C combined with objective evidence of impaired rectal evacuation, respond to pelvic floor biofeedback therapy rather than to laxatives. Increased perception of visceral sensations is more prevalent in IBS-C than in FC [1]. The dose and the efficacy of medications such as lubiprostone or linaclotide differ between FC and IBS-C [2]. These features suggest that idiopathic constipation is not a homogenous entity, providing the raison d'être for classifying constipation according to response to therapy.

Many patients with FC have abdominal pain, which blurs the distinction between FC and IBS-C. Indeed, in one study, approximately $90 \%$ of patients with IBS-C also had criteria for FC and about $44 \%$ of the FC patients also had criteria for IBS-C [3]. In patients with overlapping symptoms, a single diagnosis is only possible because the Rome criteria require patients who have symptoms of IBS-C and

Adil E. Bharucha

bharucha.adil@mayo.edu

1 Division of Gastroenterology and Hepatology, College of Medicine, Mayo Clinic, Clinical and Enteric

Neuroscience Translational and Epidemiological Research Program (C.E.N.T.E.R.), 200 First St. S.W, Rochester, MN 55905, USA
FC to be designated as IBS-C and not as FC. Moreover, it can be challenging to distinguish between FC and IBS-C in clinical practice [4] because many constipated patients who have infrequent and hard stools even in the absence of abdominal pain are puzzled when asked to relate the hardness and frequency of their bowel movements with the presence of abdominal pain. Lastly, in approximately one-third of patients, symptoms shift over time from CC to IBS-C and vice versa [3]. Hence, the current Rome system for classifying chronic constipation as FC and IBS-C appears to lack specificity and reproducibility; a superior approach is necessary.

Beginning with a small case series from the pre-Rome era, several studies in the community and in clinical practice have used abdominal pain to characterize constipated patients as painful or painless constipation (Table 1). ${ }^{1}$ In the original paper, patients with painful constipation reported more disability, somatic symptoms, and urinary urgency than those with painless constipation; painful constipation resembled IBS-C rather than FC [6]. Remarkably, similar differences between painful and mild pain constipation were observed across studies even though the definition of abdominal pain varied among these studies (Table 1) [7].

Continuing this theme, a meticulous study by Bouchoucha and colleagues in this issue of Digestive Diseases and Sciences not only evaluated symptoms, anxiety, and depression, but also colonic transit and anorectal manometry in 546 consecutive constipated patients referred to a tertiary center [8]. Of these 546 patients, 301 (53\%) and 245 (47\%), respectively, were classified as having FC and IBS-C. Based on the revised classification, $316(58 \%)$ had "mild pain" and

\footnotetext{
${ }^{1}$ Unlike the Rome criteria for IBS-C, the criteria for painful and painless constipation do not include the relationship between abdominal pain and bowel disturbances, the impetus for which originated from a factor analysis in which abdominal discomfort was associated with loose and/or frequent stools [5]. Those symptoms, which are characteristic of diarrhea-and not constipation-predominant IBS, were then reformatted (i.e., hard instead of loose stools, less frequent rather than more frequent bowel habits) to develop the criteria for IBS-C.
} 


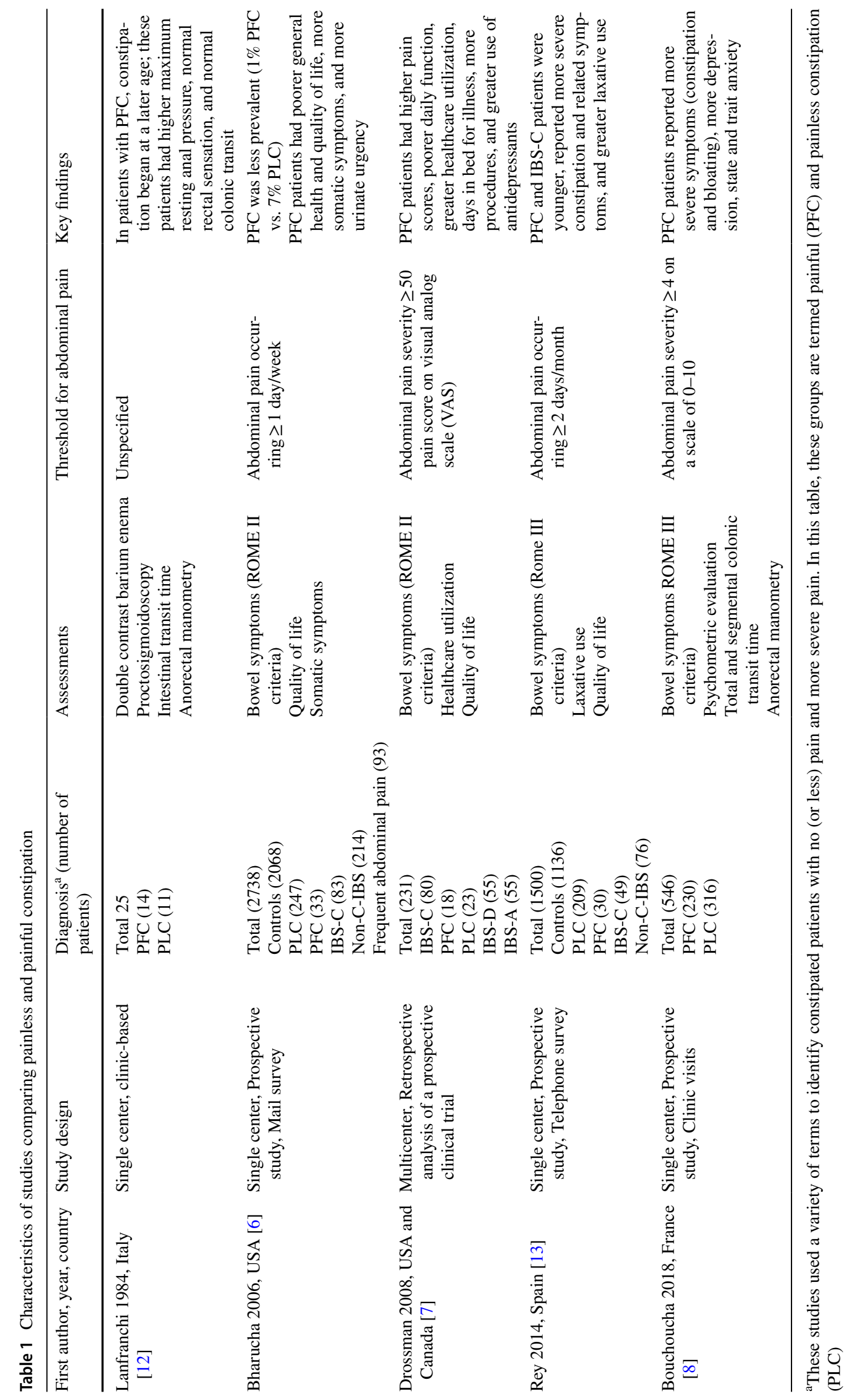




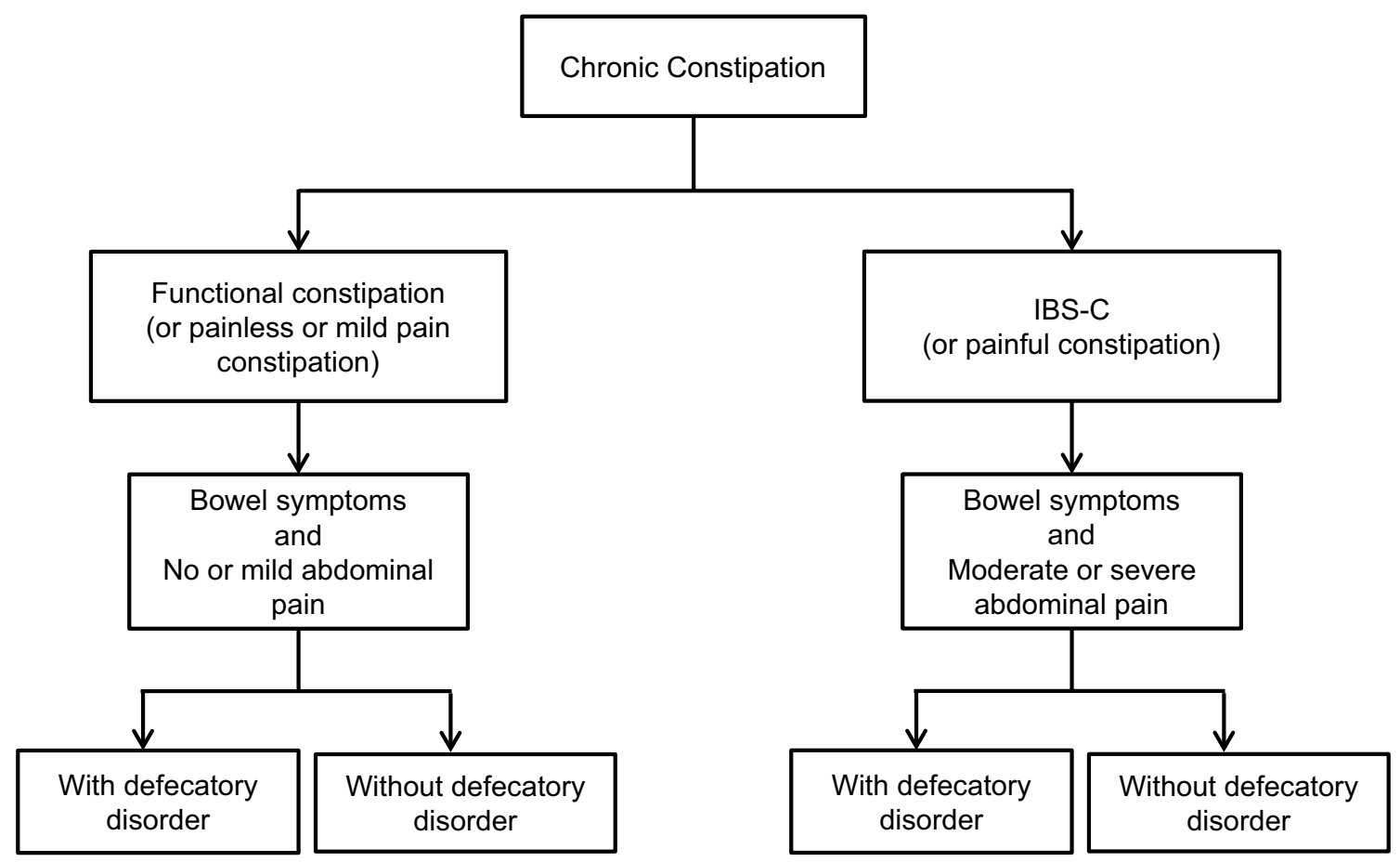

Fig. 1 Proposed revised classification of chronic constipation

230 (42\%) had "painful" constipation. Approximately $80 \%$ of patients with FC but only one-third of patients with IBS-C had "mild pain" constipation. Hence, the "mild pain" and "painful" groups predominantly corresponded to patients with FC and IBS-C, respectively. Compared to "mild pain" constipation, patients with "painful" constipation had more prominent bowel symptoms and were more likely to have upper gastrointestinal (e.g., dysphagia and dyspepsia) and anorectal symptoms, urinary and sexual symptoms, anxiety and depression, and slower rectosigmoid transit. The widespread symptoms in painful constipation may partly reflect increased perception of visceral sensations such as wall tension [1].

These findings provide the impetus for modifying the Rome criteria for constipation, which should be comprised of symptoms and objective measurements of rectal evacuation (Fig. 1). Currently, a diagnosis of DD requires symptoms of FC or IBS-C coupled with objective evidence of impaired rectal evacuation. By contrast, FC and IBS-C are diagnosed by symptoms alone; evidence of normal rectal evacuation is not required. In the Bouchoucha study, approximately $50 \%$ of patients with FC and IBS-C had evidence for impaired rectal evacuation, i.e., they actually had DD. Likewise, $50 \%$ of patients with "mild pain" and $57 \%$ with painless constipation had DD. With the increasing availability of anorectal manometric testing and the recognition that pelvic floor biofeedback therapy is superior to laxatives for $\mathrm{DD}$, all constipated patients who do not respond to simple laxatives should undergo anorectal tests to diagnose the presence of DD [2]. If necessary, additional categories (i.e., "FC unspecified" or "IBS-C unspecified") can be developed for patients in whom anorectal tests have not been performed.

Second, the symptom criteria for IBS-C should be revised, eliminating the need for criteria that solely rely on the relationship between abdominal pain and bowel disturbances. Both FC and IBS-C should be defined by bowel symptoms, respectively, without or with clinically significant abdominal pain. Ideally, the abdominal pain threshold should be easy-to-understand, universal, reproducible, should identify groups that are stable over time (i.e., minimizing switching between categories), should guide therapy such as medication dose, and should predict the response to therapy. Different abdominal pain thresholds have been used to discriminate between painless (or "mild pain") and "painful" constipation (Table 1). Rather than using an arbitrary threshold, the study by Bouchoucha used a cluster analysis to uncover the threshold that best discriminated between groups. Then, this threshold (i.e., an abdominal pain severity score of 4 on a Likert scale from 0 to 10 in the past week) was used to separate patients into two groups: "mild pain" (i.e., pain score $<4$ ) and "painful" (i.e., pain score $\geq 4$ ) constipation. This threshold score of 4 or greater on a scale of 1-10 is similar to the US Food and Drug Administration (FDA) recommended eligibility criteria in IBS-C, i.e., a weekly average of worst daily abdominal pain score of $\geq 3.0$ on a $0-10$ scale [9]. The baseline pain severity score, 
which was evaluated on a 5 -point scale (0-4), predicted the response to lubiprostone in IBS-C [10]. A baseline pain score of $\geq 3$ on an 11-point scale $(0-10)$ corresponds to a score $\geq 1.36$ on a 5-point scale [9]. The response to lubiprostone was significantly better than placebo among patients in whom the baseline abdominal pain score was $\geq 1.5$ and $\geq 2.0$, but not in patients with a score $\geq 2.5$ and $\geq 3.0$ subgroups. By contrast, linaclotide benefited patients with severe symptoms, indeed, to a greater extent than in the overall intent to treat (ITT) population [11]. This suggests that the severity of abdominal pain predicts the response to therapy. Future studies should determine the stability of "painful" and "mildpain" (or painless) constipation over time.

In summary, the study by Bouchoucha adds to the growing evidence that suggests a different approach to classifying constipation is necessary. In this instance, all roads lead to (a change in) Rome!

Funding This study was supported by Grant RO1-DK078924 to Dr. Bharucha from the National Institutes of Health (NIH).

Author's contribution AEB and MS wrote the paper and approved the final version of this manuscript.

\section{Compliance with ethical standards}

Conflict of interest The authors declare that they have no conflict of interest.

\section{References}

1. Whitehead WE, Palsson OS, Simren M. Biomarkers to distinguish functional constipation from irritable bowel syndrome with constipation. Neurogastroenterol Motil. 2016;28:783-792.

2. Bharucha AE, Chakraborty S, Sletten CD. Common functional gastroenterological disorders associated with abdominal pain. Mayo Clinic Proc. 2016;91:1118-1132.
3. Wong RK, Palsson OS, Turner MJ, et al. Inability of the Rome III criteria to distinguish functional constipation from constipation-subtype irritable bowel syndrome. Am J Gastroenterol. 2010;105:2228-2234.

4. Cremonini F, Lembo A. IBS with constipation, functional constipation, painful and non-painful constipation: e Pluribus...Plures? Am J Gastroenterol. 2014;109:885-886.

5. Whitehead WE, Crowell MD, Bosmajian L, et al. Existence of irritable bowel syndrome supported by factor analysis of symptoms in two community samples. Gastroenterology. 1990;98:336-340.

6. Bharucha AE, Locke GR, Zinsmeister AR, et al. Differences between painless and painful constipation among community women. Am J Gastroenterol. 2006;101:604-612.

7. Drossman DA, Morris C, Hu Y, et al. Further characterization of painful constipation (PC): clinical features over one year and comparison with IBS. J Clin Gastroenterol. 2008;42:1080-1088.

8. Bouchoucha M, Devroede G, Mary F, Bon C, Bejou B, Benamouzig R. Painful or mild-pain constipation? A clinically useful alternative to classification as irritable bowel syndrome with constipation versus functional constipation. Dig Dis Sci. (Epub ahead of print). https://doi.org/10.1007/s10620-018-4995-6.

9. Administration UFaD. Guidance for industry irritable bowel syndrome-clinical evaluation of drugs for treatment. In: Services UDoHaH, ed. Rockville, MD2012.

10. Chang L, Chey WD, Drossman D, et al. Effects of baseline abdominal pain and bloating on response to lubiprostone in patients with irritable bowel syndrome with constipation. Aliment Pharmacol Ther. 2016;44:1114-1122.

11. Rao SS, Quigley EM, Shiff SJ, et al. Effect of linaclotide on severe abdominal symptoms in patients with irritable bowel syndrome with constipation. Clin Gastroenterol Hepatol. 2014;12:616-623.

12. Lanfranchi GA, Bazzocchi G, Brignola C, Campieri M, Labo G. Different patterns of intestinal transit time and anorectal motility in painful and painless chronic constipation. Gut. 1984;25:1352-1357.

13. Rey E, Balboa A, Mearin F. Chronic constipation, irritable bowel syndrome with constipation and constipation with pain/ discomfort: similarities and differences. Am J Gastroenterol. 2014;109:876-884. 